\title{
Desafíos ambientales para la construcción de un postconflicto sostenible ${ }^{1}$
}

\section{Environmental challenges for the construction of a sustainable post-conflict}

\author{
Autores: Clay Said Sotelo Aragón² \\ Leticia Elena Hundek Pichón ${ }^{3}$
}

Correspondencia: claysotelo96@outlook.com, leticiahundek@hotmail.com

\section{RESUMEN}

Este artículo de investigación da cuenta de un estudio teórico y normativo que señala el ambiente como un tema trascendental en el marco del conflicto armado y del postconflicto en Colombia. Describiendo la alta relevancia ambiental que tienen las zonas más cercanas al conflicto armado como también las problemáticas ambientales consecuencia del mismo para exponer los retos y desafíos propuestos para la preservación y protección del ambiente durante el postconflicto. Sugiriendo así, los procesos investigativos como desafío y propuesta para el estudio y la búsqueda de modelos de sostenibilidad y productividad económica para el desarrollo durante el postconflicto en razón de la importancia que representa éste en el conglomerado social más cercano al conflicto.

\section{Palabras clave:}

Conflicto armado, Desafíos ambientales, Paradoja ambiental, Relevancia ambiental, Desarrollo sostenible.

\footnotetext{
${ }^{1}$ Articulo resultado de investigación del proyecto "Problemática ambiental en el marco del conflicto armado en Colombia", presentado en el X ENCUENTRO DE INVESTIGACIÓN JURÍDICA Y SOCIOJURÍDICA NODO CARIBE 2016.

-Ponencia Capítulo del Proyecto de Grado: "Investigación: propuesta en los desafíos ambientales para la construcción de un postconflicto sostenible".

-Ponencia base del Ensayo: "Desafíos ambientales para la construcción de un postconflicto sostenible", presentado en la VIII CONVOCATORIA DE ENSAYOS - Universidad Libre 2017.

${ }^{2}$ Estudiante de IX semestre del Programa de Derecho de la Universidad del Atlántico, miembro del semillero de investigación KALO, adscrito al Grupo INVIUS Categoría A COLCIENCIAS. Diplomado en Gestión de Paz y Postconflicto de la Corporación Universitaria de la Costa (CUC). Investigador en desarrollo, territorio, ambiente y análisis político. Correo electrónico: claysotelo96@outlook.com

${ }^{3}$ Socióloga de la Universidad Autónoma del Caribe, Magistrado en Estudios Político Económicos de la Universidad del Norte, Docente investigadora, PAR Académico MEN, Vice Presidenta del Comité de Ética de Investigación Universidad del Atlántico, Metodología de pregrado y posgrado, Coaching En Formación Para Educadores, Metodología del Centro de investigaciones Socio Jurídica "Luis Eduardo Nieto Arteta Universidad del Atlántico, Coordinadora General de Semilleros de Investigación de la Universidad del Atlántico. Correo electrónico: leticiahundek@hotmail.com
} 


\begin{abstract}
This paper presents a theoretical and normative study that indicates the environment as a transcendental topic in the context of armed conflict and postconflict in Colombia. It describes the high environmental relevance of the areas closest to the armed conflict as well as the environmental problems resulting from it, and in that order, sets out the challenges and challenges proposed for the preservation and protection of the environment during post-conflict. In turn, the research processes are challenged and proposed for the study and search for models of sustainability and economic productivity for development in post-conflict, taking into account the prevailing need and importance that it represents in the social conglomerate closest to the conflict.
\end{abstract}

\title{
Key words:
}

Armed conflict, Environmental Challenges, Environmental paradox, Environmental relevance, Sustainable development.

\section{Introducción}

Colombia se encuentra en un estado en el que se empiezan a percibir cambios trascendentales después de un largo periodo de conflictos armados por los que ha atravesado el país, paulatinamente llegando a su fin. De acuerdo a la historia estos conflictos tuvieron orígenes políticos, ideológicos, económicos y sociales, sin embargo existen casos en los que la disputa por la tierra y el control de los recursos naturales los generaron (Guáqueta, 2002).

Entre el Estado y grupos al margen de la Ley se han llevado a cabo procesos de paz muy importantes para Colombia y la comunidad internacional, producto de ello encontramos, la Ley de Justicia y paz, con la cual el gobierno logró desarticular una de las fuentes más importantes de violencia: simplificó el campo de confrontación militar contra los grupos armados irregulares y fortaleció la presencia de la Fuerza Pública (Acevedo, 2008). Producto de lo anterior se dio 
la desmovilización de las AUC, esto del 25 de noviembre de 2003 al 15 de agosto de 2006 (VerdadAbierta.com, s/f)

Otro avance en la terminación de conflictos armados se encuentra en el Acuerdo de Paz para la Terminación del Conflicto y la Construcción de Paz Estable y Duradera, en el cual las FARC-EP y el Gobierno de Colombia se han trazado como meta la finalización del conflicto armado interno. Actualmente, se encuentra en su fase de implementación, sin embargo, es notorio su avance de acuerdo a los objetivos trazados para la consolidación de sus fines.

De acuerdo a la serie de políticas que ha impulsado el Gobierno Nacional en aras de alcanzar el fin del conflicto armado e impulsar el desarrollo político, económico y social es menester señalar que dentro de estas; existen agendas, planes, programas y proyectos que deben llevarse a cabo en el periodo de transición del fin del conflicto; y posterior al fin del conflicto en el marco del postconflicto, teniendo en cuenta estudios e informes ajustados a la realidad de la nación.

Colombia a lo largo de los años ha estado inmersa en un conflicto armado con características especiales que lo diferencian de cualquier otro en el mundo. En un aspecto particular señalamos que esas connotaciones representan en el postconflicto desafíos y retos que requieren desde diversos enfoques reestructurar el ordenamiento jurídico, impulsar políticas, planes, programas y proyectos que lleven consigo los aspectos más propicios para el desarrollo del Estado en todas sus esferas.

El conflicto armado en Colombia se ha presentado mayormente en las zonas rurales ${ }^{4}$ del territorio nacional, esto representa una característica especial de acuerdo a la forma como se ha desarrollado el conflicto ya que Colombia es el segundo país con mayor biodiversidad del planeta después de Brasil (Celín,

\footnotetext{
${ }^{4}$ Las zonas rurales en Colombia están compuestas por bosques, selvas, llanos y sistemas montañosos, lo que constituye variadas características geográficas.
} 
2007), de esta forma se infiere que el conflicto armado en Colombia posee una alta relevancia ambiental; por lo que es necesario que a través de estudios e informes se determinen los impactos ambientales que ha dejado.

El Estado debe establecer métodos para la recuperación y preservación de las zonas de postconflicto, garantizando el cumplimiento de sus fines y lo previsto en la Ley. La ONU ha propuesto unas recomendaciones en material de desarrollo rural y sostenibilidad, ordenamiento territorial y fortalecimiento de la economía, adecuadas a la realidad social y ambiental que presenta el postconflicto en Colombia.

Para efectos de esta investigación, se considera que de acuerdo la normatividad y el contexto, es necesario complementar estas recomendaciones a través de una propuesta investigativa en la que se analicen los desafíos ambientales que tiene el postconflicto y los territorios de alta relevancia ambiental. Lo anterior con miras a alcanzar mayores índices de sostenibilidad y reconocimiento del ambiente, pues indiscutiblemente los territorios megadiversos deben preservarse.

A través de esta ponencia se presenta un análisis de las paradojas y contradicciones ambientales que existen frente al conflicto, los desafíos medioambientales que tiene el postconflicto por superar, y cómo los procesos investigativos del entorno y el medio botánico natural incidirían en el desarrollo sostenible y el fortalecimiento económico con actividades adecuadas al entorno de postconflicto.

\section{Metodología}

Esta ponencia responde desde la perspectiva metodológica a una investigación socio-jurídica al resaltar los vínculos entre el ordenamiento jurídico colombiano y normas internacionales con el bien jurídico: ambiente, bajo un tipo de investigación descriptivo señalando las características del fenómeno estudiado. 
Vol.9, No 1. Diciembre de 2017 pp. 53-75

Con un método de investigación teórico, teniendo en cuenta las sucesivas aproximaciones al fenómeno estudiado. Con fuentes de información secundarias y técnicas de análisis de contenido cuantitativo para presentar una postura con respecto a la protección jurídica al ambiente en el postconflicto.

\section{Resultados y discusiones.}

\section{La paradoja ambiental del conflicto armado.}

En un proceso de paz que ha transcurrido por unos años y se encuentra en su fase de implementación, en el cual los acuerdos alcanzados entre el Gobierno y las FARC en la mesa de diálogos fueron tocados diversos temas en el que se destacan los cultivos ilícitos, encontrándose en proceso de materialización. Es menester, a partir de allí señalar que el Gobierno Nacional y diferentes organismos internacionales, entre ellos la Organización de las Naciones Unidas han tomado medidas frente al uso, manejo y erradicación de cultivos ilícitos en respuesta a la lucha contra el narcotráfico.

No obstante, un punto a lugar relegado, es el de protección natural y recuperación de los escenarios devastados por el conflicto, a través de políticas de sostenibilidad o la aplicación de la normatividad en materia. En ese orden de ideas, se ve implícita una paradoja ambiental en el conflicto armado: por un lado, frente a la relevancia ambiental que posee entre el 90 y $100 \%$ de las poblaciones implicadas en el conflicto y por otro, el tipo de actividades productivas que se deben llevar a cabo a partir de la Reforma Rural Integral que expone el Acuerdo de paz ${ }^{5}$.

\footnotetext{
${ }^{5}$ Desarrollo integral del campo: el desarrollo integral del campo depende de un adecuado balance entre las diferentes formas de producción existentes -agricultura familiar, agroindustria, turismo, agricultura comercial de escala-; de la competitividad y de la necesidad de promover y fomentar la inversión en el campo con visión empresarial y fines productivos como condición para su desarrollo; y de la promoción y fomento, en condiciones de equidad, de encadenamientos de la pequeña producción rural con otros modelos de
} 
Es pertinente mencionar la necesidad de regulación normativa internacional para la protección y preservación del ambiente, pues, a falta de regulación internacional en materia de gestión compartida de recursos a escala global se presenta un factor adicional en el grado de conflictividad (Chesnot, 1992), dejando así la posibilidad a la generación de nuevos conflictos. Lo anterior en relación al conflicto armado colombiano, en el cual uno de sus detonantes ha sido el uso y tenencia de la tierra representada en una inequidad de recursos y un enfoque agrario (Le Grand Catherine, 1988) .

\section{Las consideraciones de la ONU para un postconflicto sostenible en el marco normativo colombiano.}

Teniendo en cuenta que los conflictos ambientales a nivel global se mantienen siempre dentro del marco político, económico y social del Estado; en el caso concreto de Colombia, en un escenario de postconflicto es necesario establecer parámetros de reparación y preservación del medio ambiente. De acuerdo a esto, la ONU en trabajo conjunto con Cooperación Alemana ha propuesto a través de un informe presentar las consideraciones ambientales para la construcción de una paz territorial, duradera y sostenible, a su vez ligada a la normatividad colombiana. (ONU, 2014).

Dentro del marco legal colombiano se señala un largo periodo de legislaciones que ofrecen una línea de protección, a groso modo, el Decreto 2811 de 1974, mediante la cual se estableció el Código de Recursos Naturales hace énfasis en la prevención y control del ambiente como patrimonio común. Por tanto acarrea una obligación hacia el Estado y los particulares su protección y preservación. No obstante, resultó necesario crear una entidad garante de la organización, regulación, control y vigilancia en relación con la protección ambiental.

producción, que podrán ser verticales u horizontales y en diferente escala. En todo caso se apoyará y protegerá la economía campesina, familiar y comunitaria procurando su desarrollo y fortalecimiento.

${ }^{6}$ gran parte del conflicto que se inicia con los años 40 surge de la lucha por la posesión de la tierra cuando los colonos independientes se convirtieron, por la fuerza, en arrendatarios y jornaleros. 
Por lo que es proferida la Ley 99 de 1993, que crea el Ministerio del Medio Ambiente, se reordena el Sector Público encargado de la gestión y conservación del medio ambiente y los recursos naturales renovables, se organiza el Sistema Nacional Ambiental, SINA, y se dictan otras disposiciones para contrarrestar la degradación ambiental que se venía presentando a pasos agigantados sin control alguno. Lo anterior en aras de priorizar la aplicabilidad del Código de Recursos Naturales, impulsando en su ejercicio control y vigilancia en materia ambiental.

Dentro de la normatividad también se destaca la Ley 115 de 1994, Por la cual se expide la Ley general de educación. Si bien esta regula temas referentes a educación, servicios educativos, entre otros, es preciso hacer énfasis en su artículo 5 numeral $10^{\circ}$ en el que se señala como fin de la educación: La adquisición de una conciencia para la conservación, protección y mejoramiento del medio ambiente, de la calidad de la vida, del uso racional de los recursos naturales, de la prevención de desastres, dentro de una cultura ecológica y del riesgo y de la defensa del patrimonio cultural de la nación? ${ }^{7}$.

\section{El sentido Constitucional - Bloque de constitucionalidad del ambiente}

Por su parte, la Constitución Política de 1991, establece una plataforma de protección revestida de herramientas jurídicas más agiles. A través de las acciones constitucionales, entre éstas la Acción de Tutela y la Acción Popular, enunciadas en los artículos 86 y 88 respectivamente, otorgaron al conglomerado social el deber y la facultad de velar por la preservación del ambiente, así como exigirlos en caso de vulneración contra los derechos individuales y colectivos.

La Carta Política se destaca por ser una Constitución verde. A lo largo de su cuerpo normativo señala preceptos en favor del medio ambiente, esto se apoya en el Preámbulo y los artículos 2, 8, 49, 58, 66, 67, 78, 79, 80, 86, 88, 95, 268, 277, 289, 300,302, 310, 313, 317, 330, 340 y 366 (Gómez, 2007), que reviste de calidades y responsabilidades al Estado de ser garante y ofrecer un ambiente

\footnotetext{
${ }^{7}$ La ley 115 de 1994 señala las normas generales para regular el Servicio Público de la educación que cumple una función social acorde con las necesidades e intereses de las personas, de la familia y de la sociedad, bajo la anterior premisa se amolda la educación a la sensibilización y preservación del medio ambiente.
} 
sano, para ello, debe legislar, desarrollar políticas, técnicas científicas que permitan tal fin, también revisten de deberes a sus ciudadanos para el cumplimiento de lo dispuesto en la Carta.

Lo anterior, se encuentra apoyado a su vez, por diversos Tratados Internacionales y disposiciones que alrededor de esta problemática se han expedido, en este caso la ONU. Quien declaró en 2002, el 6 de noviembre como el día internacional a la prevención de la explotación del medio ambiente en los conflictos armados, lo mismo que una serie de documentos como: "Rol de los recursos naturales en materia de desarme, desmovilización y reintegración", publicada en diciembre de 2013.

Otro documento de íntima relación es: "El manejo de los recursos naturales en las situaciones de transición", los anteriores documentos hacen referencia a las políticas adecuadas para enfrentar problemáticas en asuntos medio ambientales dentro del conflicto armado y el postconflicto. También se establece la: Convención sobre prohibición de utilizar técnicas de modificación ambiental con fines militares y otros fines ambientales, como también la Convención ENMOD, aprobada por las naciones unidas (United Nations, 1976).

Resulta necesario manifestar que Colombia se ha caracterizado durante años por acogerse a gran variedad de tratados internacionales en materia ambiental. Es así como la Convención de Estocolmo 1972, continuando con la Convención de Rio 92 y subsidiarios a éstos, los relativos al cambio climático, el Protocolo de Kyoto, 15 tratados bilaterales y 70 multilaterales. Todos ellos suscritos y en la mayoría de los casos ratificados por nuestro país, de acuerdo con lo establecido en la Biblioteca Virtual de Tratados de la Cancillería Colombiana (Lastra, 2015).

En Colombia, la jurisprudencia ha direccionado también el ordenamiento jurídico a la protección natural y recuperación de los escenarios del conflicto en 
sentencias T-528 de $1992^{8}$, C-293 de $2002^{9}$, C-703 de $2010^{10}$, T-204 de $2014^{11}$, donde la Corte Constitucional describe el medio ambiente como bien jurídico y señala Los principios que guían el derecho ambiental, así como la necesidad de que la Constitución Nacional regule de los ejes en los que se desarrolla la relación entre: El medio ambiente, el conglomerado social, el Estado, los particulares y los medios de producción.

Ahora bien, es preciso por de la extensa normatividad en materia ambiental en Colombia, que cada una de las normas en concordancia se estructuren o estén acordes dentro del postconflicto, esto jurídica y políticamente hablando. Cabe anotar que el país ha reestructurado su marco legal y normativo, asumido iniciativas de políticas, fortalecido su capacidad para proteger y manejar tanto sus recursos naturales como su calidad ambiental, y ha creado un sistema de Parques Nacionales y Reservas Forestales que abarca más de una cuarta parte del país.

Sin embargo también han habido retrocesos en esta materia debido al interés del Gobierno nacional por atraer inversión extranjera sin importar las consecuencias para que el país se inserte en el mercado global (Molina Orjuela, 2016), como pudo notarse con el otorgamiento de licencias ambientales en paramos y que al respecto la sentencia C-035 de 2016 contrarrestó. Esta es solo una cara de la curiosa paradoja que rodea las relaciones entre el conflicto armado y el ambiente en Colombia (Montaño, 2015) durante el postconflicto, lo que el Gobierno ha denominado desarrollo sostenible.

De conformidad a lo anterior esto representa un desafío frente a la forma de distribución de la tierra en el territorio colombiano, así como el uso y explotación de recursos. Pero mecanismos como la consulta popular y las acciones constitucionales siguen siendo objeto de protección y preservación del ambiente,

\footnotetext{
${ }^{8}$ La política ambiental debe encontrarse guiada por principios generales de derecho.

${ }^{9}$ La carta de 1991, consagra el derecho al ambiente sano, no como un derecho constitucional fundamental, sino como un derecho y un interés constitucional de carácter colectivo.

10 "Prevención y precaución, que persiguen, como propósito último, el dotar a las respectivas autoridades de instrumentos para actuar ante la afectación, el daño, el riesgo o el peligro que enfrenta el medio ambiente, que lo comprometen gravemente, al igual que a los derechos con él relacionados".

11 "la ratio iuris de la Constitución ecológica".
} 
para atender retos en cuanto a planificación, monitoreo y control de los recursos naturales en zonas de postconflicto ricas en recursos naturales ${ }^{12}$.

Existe una clara evidencia de que estas consecuencias ambientales y sociales negativas han formado en realidad, una nueva fuente de conflicto entre una gama de actores con diferentes intereses como las empresas multinacionales, pequeñas minas tradicionales e informales, minas ilegales, instituciones gubernamentales, comunidades locales, organizaciones no gubernamentales y otros actores de la sociedad civil. De hecho, el propio Gobierno colombiano reconoce esto en el Plan Nacional de Desarrollo 2014-2018 (Lowe, 2016).

La diversidad natural y cultural de la nación forma parte del patrimonio fundamental en la construcción de paz y país con el fin del conflicto. Por ello, reconocer que los ecosistemas son en estricto sentido socio-ecosistemas, usados y transformados de forma sostenible por sociedades concretas, debería orientar el postconflicto desde criterios de reparación, compensación y retribución a esas sociedades, promoviendo sus prácticas ambientales y desincentivando el mal manejo de los recursos naturales.

Con la finalidad de propiciar un correcto ordenamiento jurídico-político de conformidad a la normatividad nacional e internacional, la necesidad que surge frente al desarrollo y la ejecución de desafíos en materia ambiental para la construcción de paz en el marco del postconflicto. La prevalencia que debe adquirir se presenta a través de Planes de Ordenamiento Territorial y Planes de Ordenamiento Minero ${ }^{13}$, de conformidad la Ley 1454 de 2011 "LOOT"14, fórmulas y mecanismos en aras de preservar y proteger la mega biodiversidad colombiana es realmente necesario.

\footnotetext{
${ }^{12}$ Las poblaciones implicadas en el conflicto tienen una alta relevancia ambiental de entre el 90 y $100 \%$ en recursos, fauna y flora.

${ }^{13}$ Plan Nacional de Ordenamiento Minero - PNOM.

${ }^{14}$ En una variedad de Artículos como el $3 \# 15,6,9,15,16$, entre otros, se puede encontrar componentes ambientales frente al modo de organización y ordenamiento territorial.
} 
Es menester, proponer a través del desarrollo, los desafíos ambientales en el postconflicto. Crear una relación de los factores ambientales existentes, la manera en la que se ha llevado el conflicto y se pretende llevar en el postconflicto, dado que el medio ambiente a la fecha no ha sido objeto de relevancia por el Gobierno en el conflicto. No se habla de seguridad ambiental tal como se habla de seguridad ciudadana y nacional (democrática) o de seguridad económica. No ha entrado como tema de alta política en la agenda nacional a pesar de ser un asunto muy sensible para el Estado colombiano (LAVAUX, 2004).

\section{Los desafíos medioambientales en el postconflicto}

\section{Generalidades}

Colombia es uno de los países más importantes del planeta en materia ambiental; Presenta una alta variedad biológica, por lo que es considerado el segundo país megadiverso del mundo, albergando cerca del $10 \%$ de biodiversidad en el planeta. Este considerable porcentaje en tan sólo el $0.7 \%$ de la superficie terrestre (ROMERO M, 2008). La gran variedad de ecosistemas y biomas existentes contiene una cantidad importante de endemismos o de concentración de especies naturales, que sólo es superada por Brasil, con un territorio siete veces mayor que el de Colombia (Molina, 2011).

Sin embargo, Colombia ha sido mencionada por diferentes estamentos académicos, políticos y jurídicos nacionales e internacionales debido al conflicto armado prevalente, que ha ocasionado serias afectaciones a la gran riqueza ambiental que posee, en la medida que el principal escenario del conflicto han sido las áreas rurales, selváticas y montañosas del territorio nacional; donde se concentra toda esa megabiodiversidad, la cual se ha degradado significativamente.

Años de lucha constante han generado una de las más importantes y notorias catástrofes ambientales del mundo. No en vano nos hemos habituado a 
las noticias referentes a voladuras de oleoductos, deforestación de grandes extensiones de territorio por cultivos ilícitos, aniquilación de zonas protegidas, contaminación de grandes extensiones de selva y bosques por pesticidas en el vano intento de erradicación de cultivos ilícitos y actualmente un fenómeno de rapiña de territorios por causa de la minería (Lastra, 2015).

Dentro de los daños que se producen al ambiente la gran mayoría resultan irreversibles y de gran dificultad para subsanar estas zonas debido a que su recuperación resulta demasiado lenta frente a las necesidades que requiere el hombre y el medio (fauna y flora). Claro ejemplo de lo anterior está en la producción de narcóticos y cultivos ilícitos, los cuales se encuentran en zonas montañosas y selváticas del país de difícil acceso para la población civil, pero donde existe todo un ecosistema megadiverso.

Para que logre ser útil el terreno, bosques y selvas son quemados antes de sembrar coca, marihuana o amapola. En consecuencia a la poca fertilidad y la necesidad de evadir a las autoridades, los cultivos ilícitos son abandonados después de dos o tres siembras y se repite el mismo ciclo en otra posición. Quedando un grave daño realizado en el que volver a recuperar este espacio natural tarda más de 50 años (Rodríguez, 2003). Esta práctica inconsciente ha acelerado la deforestación y destruido recursos madereros que podrían estar disponibles para un uso sostenible de la tierra selvática.

Se necesita mayor comprensión frente a esta problemática, pues tanto el Gobierno Nacional como los gobiernos extranjeros deben entender que Colombia ha padecido una violencia extrema y singular que tiene mucho fundamento con el narcotráfico (Acevedo, 2008), pero también de éste se desprenden problemáticas inconmensurables, entre estas, se precisa que por un lado encontramos los enfrentamientos entre grupos armados en zonas rurales y urbanas por el control del "negocio" y por otro la gran problemática del conflicto ambiental.

Los conflictos ambientales se manifiestan a manera de conflictos políticos, sociales, económicos, culturales, étnicos, religiosos y territoriales o como 
conflictos por recursos naturales de interés nacional. Son conflictos tradicionales inducidos por una situación de degradación ambiental causada por una sobreutilización (o mala administración) de los recursos, contaminaciones o empobrecimiento del espacio vital (LAVAUX, 2004), efecto de lo anterior, presenta incidencia en los niveles de pobreza y acceso a servicios básicos (Kang, 2014), y factores como la alteración y deforestación del medio ambiente.

Dentro del marco del conflicto armado surgen conflictos ambientales por cuanto encontramos cerca de 7 millones de hectáreas en el país que se encuentran involucradas actualmente. De las cuales, en gran medida han sido empleadas para fines como la minería ilegal, responsable de daños irreparables; y la alteración y deforestación del medio botánico natural para la producción de cultivos ilícitos y su posterior fumigación para la erradicación de estos por parte del Gobierno Nacional con el químico denominado: glifosato, lo que constituye graves deterioros a la fauna y flora.

Consecuencia del conflicto ambiental encontramos en los cultivos ilícitos una afectación al marco social, económico y cultural de la población rural, pues, dentro del marco de la deforestación se calcula que para sembrar una hectárea de amapola se deforestan dos y media de bosque andino y cuatro de selva (Osorio, 2003), de la anterior premisa se logra calcular alrededor de seis millones de hectáreas deforestadas en los últimos 20 años, producto de la convergencia del narcotráfico y grupos armados al margen de la Ley.

Toda esa defaunación está causando una degradación de muchos de los ecosistemas boscosos del país debido, entre otras, a la desaparición o disminución de dispersores críticos de las semillas vitales para la reproducción de la flora (Becerra, 2004). Para frenar esto, es necesaria la intervención del Estado para la recuperación y preservación del medio ambiente, como también prever que de ello depende la subsistencia de generaciones venideras. Se requieren fijar 
Vol.9, No 1. Diciembre de 2017 pp. 53-75

unos parámetros, actividades, planes y programas para hacer frente a los efectos que ya son notorios.

\section{Los desafíos ambientales}

Entonces, ¿Cuáles son los desafíos que se deben prever durante el postconflicto en un país que ha sufrido el flagelo de la guerra en casi todas las esferas incluso ambientalmente hablando? En respuesta a ésta incógnita el ministerio del Medio Ambiente, en alianza con cinco de las agencias más importantes de Naciones Unidas (PNUD, FAO, OIM, Pnuma Y Unodc) y la Cooperación Alemana, realizando el ejercicio se encontraron sorprendentes resultados (ONU, 2014).

En el proyecto llevado a cabo se estudiaron 125 municipios prioritarios para programas en el marco del postconflicto, teniendo en cuenta el mapa de la poblaciones con mayor índice de violencia en Colombia, a su vez, con los datos del mapa geológico de Ingeominas, el mapa de ecosistemas colombiano, el Estudio nacional del agua, de Ideam y el Estudio de los conflictos de uso del territorio colombiano, del Instituto Geográfico Agustín Codazzi.

Se pudo establecer que estas zonas tienen altísima relevancia ambiental y que en lo que respecta a la implementación de la Reforma Rural Integral que plantea el Acuerdo de paz es notoria una gran cantidad de retos. Esto basado en la realidad de aplicación y manejo de los recursos naturales en Colombia que comprende falencias en presencia del uso de zonas de reserva forestal previsto lo anterior en la legislación colombiana ${ }^{15}$.

Es pertinente la promoción de actividades productivas que vayan realmente acorde a lo que requiere el medio como modelos de desarrollo agrícola, fortalecimiento del campo y recuperación de actividades sostenibles, enfatizando en el correcto uso del suelo y materia prima disponible. Lo anterior, dejando a un

\footnotetext{
${ }^{15}$ La legislación colombiana regula el uso producto de diferentes actividades en zonas de reserva forestal a través de la Ley 2 de 1959, el Decreto 2811 de 1974, el Decreto 1608 de 1978, el Decreto 2372 de 2010 y la Sentencia C-030 de 2008 entre otras disposiciones normativas afines.
} 
lado las actividades nocivas para el medio ambiente como la ganadería en grandes proporciones y la explotación minera, lo que de acuerdo a los planteamientos presentados anteriormente en esta ponencia representa conflictos ambientales.

Otro de las proyecciones que sostiene la ONU como un desafío medio ambiental en el postconflicto, es la necesidad de una institucionalidad, enfocada en la protección y preservación de territorios megadiversos, de una forma eficiente y con capacidad de respuesta rápida (desde lo regional y lo nacional) capaz de atender los retos que impone el crecimiento poblacional y estructural de los territorios planificación, monitoreo y control.

Es necesaria la inclusión dentro de los Planes de Ordenamiento Territorial la organización de los territorios de alta relevancia ambiental en el postconflicto, de modo que exista un correcto manejo de éste por parte de las autoridades, así como el sector privado e industrial. No obstante, es necesario priorizar los territorios que la Ley dispone, tales como: territorios indígenas, ancestrales, colectivos y afrocolombianos. Lo anterior dando cumplimiento a lo enunciado en la Ley 1454 de 2011.

Un punto que resulta controversial y sensible al momento hacer referencia es el tema de acceso a la tierra. La historia del país ha estado marcada por una debilidad institucional que, entre otros aspectos, se manifiesta en la indefinición de los derechos de propiedad, con efectos tales como la agudización de los desequilibrios sociales y los reiterados estallidos de violencia. Entre ellos la lucha por el poder territorial entre grupos armados ilegales exacerbada por el narcotráfico (Albán, 2011).

En Colombia la tierra ha sido un detonante en medio del conflicto armado, bien sea por el control de territorios o por la insaciable sed de poder económico de grupos al margen de la Ley. En ese orden de ideas es necesaria la democratización del acceso a la tierra; claro está, con la promoción de estrategias y aprovechamiento de la biodiversidad de manera sostenible. A su vez, resulta 
Vol.9, No 1. Diciembre de 2017 pp. 53-75

necesario un mecanismo de financiación para ello, lo cual sería a través de impuestos prediales, tasas por uso de agua y servicios ambientales.

\section{La Investigación como desafío en el postconflicto}

Los tres lineamientos anteriormente planteados representan ideas que el estudio en mención presenta con miras al postconflicto de nuestro país. Sin embargo, diversos estudios académicos, políticos, de acuerdo al panorama jurídico presentan retos y desafíos de carácter ambiental que siguen trascendiendo dentro del conflicto armado, estos, requieren para el postconflicto diferentes alternativas, acordes a la realidad social, política y económica de los sectores de alta relevancia ambiental involucrados en el proceso de postconflicto.

Por esta razón se propone la investigación como otro desafío en el postconflicto. Lo cual resulta necesario ya que gran cantidad de territorios, producto del conflicto se han mantenido intactos y sin mayor impacto por parte del hombre. Al respecto, señala Brigitte Baptiste, directora del Instituto Alexander Von Humbold: "Una de las limitaciones es acceder a varias regiones, porque en ciertas condiciones se podía ir y tomar muestras de afán, pero realmente hacer modelos científicos sobre el manejo del bosque o de los ecosistemas era prácticamente imposible" (Gutiérrez, 2016) .

En el mismo sentido se requiere estudiar y analizar los diferentes comportamientos de los territorios degradados por el conflicto con procesos investigativos, ya que de éste modo se puede delimitar las necesidades y problemáticas ambientales para que el Gobierno Nacional y los entes territoriales tomen medidas eficaces para los modelos de desarrollo sostenible y ordenamiento territorial, pero no queda simplemente ahí.

Con mayor número de estudios e investigación en localizaciones del territorio nacional donde el conflicto ha 0 no ha permitido descubrir toda esa biodiversidad, es muy seguro que las recomendaciones de la ONU tendrán mayor aplicabilidad y eficacia, esto, debido a que es absolutamente necesario para llevar a cabo Planes de Ordenamiento Territorial, reformas rurales y preservación del 
Vol.9, No 1. Diciembre de 2017 pp. 53-75

medio ambiente conocer las características específicas del medio, su biota y endemismos.

La investigación como desafío ambiental requiere un análisis a la perspectiva institucional, su conducción, cómo va a representar y ser de alta relevancia el tema del medio ambiente en el postconflicto de acuerdo a la vista de los sectores políticos, para hacer frente a la legislación, impulsar políticas para la especial protección del medio ambiente, brindando a su vez reparación, cosa que muy poco se percibe dentro de los últimos acuerdos paz de los conflictos armados $^{16}$. Pero también en el mismo sentido se logra apreciar otro desafío concordante a los anteriores.

En un país que tiene más de la mitad de su superficie cubierta por bosques naturales, muchos de ellos bajo la categoría de reserva forestal, el reto más grande radica en que la construcción de paz sea una oportunidad para el desarrollo de modelos sostenibles y que éste se reconcilie con los sectores económicos en aras de la protección de valiosos recursos naturales que Colombia posee. Tal relación de modelos económicos y de desarrollo sostenible, que resulten en productividad, progreso y competitividad para las regiones sin dejar de lado el estudio de las zonas de conflicto.

Con la investigación de zonas de postconflicto, describir las necesidades para el desarrollo sostenible posibilita encontrar diferentes planes, programas y proyectos que de acuerdo al estudio del entorno, sus necesidades y problemáticas sociales se logre consolidar el desarrollo de las poblaciones aplicando modelos de sostenibilidad y productividad económica, todo orientado a alcanzar las metas que demanda el conglomerado social de nuestro Estado.

\footnotetext{
16 Del año 2003 a la fecha se han llevado a cabo procesos de paz, desmovilización y reinserción a la vida civil: con las Autodefensas Unidas de Colombiana (AUC) entre 2003 y 2006 con Justicia y Paz; y Con las Fuerzas Armadas Revolucionarias de Colombia (FARC) con el Acuerdo Final para la Terminación del Conflicto entre 2012, firmado el proceso en 2016 y en curso la implementación a la fecha 2017.
} 


\section{Conclusiones}

Se profundizó en el análisis de los diferentes componentes normativos y teóricos que hacen referencia a la preservación y protección del medio ambiente a través de los Planes de Ordenamiento Territorial, el fortalecimiento de actividades económicas en zonas de alta relevancia ambiental, la importancia de la sostenibilidad, el desarrollo a través de la utilización de recursos naturales y los preceptos económicos, políticos y sociales a que hay lugar dentro del ordenamiento jurídico.

Dentro de la investigación se logró estudiar la relación que guarda el ambiente con el conflicto armado colombiano, esto se apoya en el desencadenamiento de conflictos ambientales, por tanto representa un riesgo que se excluyan entre sí, debido a que en los procesos paz es necesario que se formulen y prevean políticas ambientales vinculadas directamente al postconflicto. Lo que es importante para la construcción de una paz estable y duradera.

En el caso de Colombia, se acentúa la problemática más allá del conflicto, se extiende a la tenencia de la tierra, las reformas rurales inequitativas y la poca presencia e intervención del Estado en asistencia, protección y control de las zonas rurales. Por tal, es menester tener un enfoque frente a la sostenibilidad y el desarrollo de las poblaciones víctimas del conflicto que habitan en zonas que representan una alta riqueza natural y relevancia ambiental.

Un aspecto para estudiar en ésta temática radica en las acciones constitucionales frente a la forma de distribución de la tierra en las zonas rurales y la explotación de recursos naturales. Del mismo modo, se sugiere que dentro de ese mismo marco se prioricen los procesos investigativos de descripción y análisis de las problemáticas ambientales, así como el entorno y la viabilidad de procesos de desarrollo sostenible y la integración de los factores económicos en las zonas 
de transición; lo cual es de gran importancia dado que en la actualidad se perciben considerables cambios debido al postconflicto.

Actualmente, Colombia se encuentra en la búsqueda de la consolidación de la paz, enfocada no solamente en la armonía entre todo el andamiaje del Estado, sino con la convicción de que es posible alcanzar nuevos objetivos como la vinculación directa al ambiente como partícipe del conflicto lo cual ha llevado a alarmantes niveles de degradación, acompañado del silencio cómplice por parte del Gobierno Nacional ante la poca intervención desarrollada por parte de sus instituciones a lo que es, un tema de alta relevancia, siendo que el postconflicto está mayoritariamente en zonas rurales y zonas de alta relevancia ambiental.

Es necesario analizar las vías por las cuales los conflictos armados han tenido grandes fracasos frente a los procesos de paz por los que han atravesado, y es que se debe entender que "el logro de la paz no es la simple negociación de la confrontación armada, sino también el desarrollo de un conjunto de medidas económicas, políticas y culturales con las que se logre la justicia social y se supere el sentido de la violencia en la solución de los conflictos internos" (Botero, 2008). Los procesos de paz tienen complejidades que requiere de diferentes esferas sociales, políticas, económicas y jurídicas para alcanzar un postconflicto real y duradero, es decir, el análisis completo de la estructura y funcionamiento del Estado.

En ese sentido, se presentan grandes retos referente al desarrollo de la temática, por cuanto existen diferentes interpretaciones de desarrollo sostenible, debido a que es un concepto sujeto a políticas específicas y modelos económicos, es decir, varía según el ordenamiento jurídico y el campo de aplicación por lo que se hace necesario que primordialmente se realice un estudio de la normatividad interna y generalizar la externa. En el mismo sentido se requiere de acuerdo al estudio teórico ajustarlo de acuerdo al sentido que propone la norma. 
Existe una controversia teórica toda vez que los diferentes estudios se centran en el desarrollo de políticas públicas más que al cumplimiento de los fines esenciales del Estado. Por ello el investigador debe abordar un campo específico, actualmente esta temática se encuentra en un punto en el que es posible obtener gran variedad de productos de investigación a razón de los procesos de paz en que se encuentra en curso, en Colombia. Es pertinente emplear estudios interdisciplinarios en el campo de la biología o la economía para ajustarlos a la normatividad. A su vez deseable que la información se complemente con artículos periodísticos o contenidos en blogs, dado que en su mayoría se centran en las diferentes problemáticas del contexto.

\section{Referencias.}

Acevedo, D. (2008). Qué pasó con el paramilitarismo en Colombia. En e. a.

Acevedo D, PARAPOLÍTICA - Verdades y mentiras (pág. 260.). Bogotá: Planeta Colombiana S.A.

\section{Albán, Á. (2011). REFORMA Y CONTRARREFORMA AGRARIA EN COLOMBIA. Rev.econ.inst. vol.13 no.24.}

Becerra, M. R. (17 de mayo de 2004). GUERRA Y MEDIO AMBIENTE. EL TIEMPO.

Botero, L. (2008). El sistema investigativo de la fundación Nuevo Arco Iris. En e. a. Acvedo C, Parapolítica- Verdades y mentiras (pág. 162). Bogotá: Planeta Colombiana S.A. 
Celín Navas, H. (2007). Memorias del segundo Encuentro de Profesionales Afrocolombianos y primero Afroamericano. Santiago de Cali: Universidad Santiago de Cali.

Correa, M., Jaimes, J., Cardozo, S. \& Mantilla, C. (2013). Estrategia para la disminución de violencias en la escuela en la ciudad de Bucaramanga. Revista Justicia, 18(24), 14-24. Recuperado de http://publicaciones.unisimonbolivar.edu.co/rdigital/ojs/index.php/justicia/arti cle/viewFile/136/138

Chesnot, C. (1992). Les enjeux pour Israël de la Confèrence Internationale sur l'eau au Proche Orient. Egypte/Monde Arabe, №. 10,, 10.

Gómez, F. (2007). Constitución Política de Colombia- Código Anotado, Bogotá . Bogotá: Leyer.

Guáqueta, A. (2002). Dimensiones políticas y económicas del conflicto armado en Colombia: anotaciones teóricas y empíricas. Colombia Internacional Revista $N^{\circ} 55,19-36$.

Gutiérrez, K. (19 de abril de 2016). The environment will be a priority in the postconflict. Territorio.

Kang, O. d.-w. (2014). Informe de vista a Colombia- 2014. 
Lastra, R. (2015). Degradación ambiental como consecuencia del conflicto armado en Colombia.

LAVAUX, S. (2004). Degradación ambiental y conflictos armados: las conexiones. Facultad de Ciencia Politica y Relaciones Internacionales; Documento de Investigación n;7., 27.

Le Grand Catherine. (1988). Colonización y protesta campesina en Colombia. Bogotà : Universidad Nacional.

Lowe, L. (2016). Postconflicto e impacto del modelo minero-extractivo sobre el medio ambiente. En A. M. Valdivieso, Política Exterior ColombianaEscenarios y desafíos en el postconflicto (págs. 226, 227.). Bogotà,: Pontificia Universidad Javeriana: Fundaciòn Konrad Adenauer,.

Molina Orjuela, D. E. (2016). Gobernanza ambiental y postconflicto en Colombia. VIII Congreso de Relaciones Internacionales, (pág. 35). La Plata.

Molina, C. D. (2011). ECOTURISMO EN COLOMBIA: UNA RESPUESTA A NUESTRA INVALUABLE RIQUEZA NATURAL. Revista de investigación en turismo y desarrollo local,. 
Montaño, E. (24 de 08 de 2015). ¿Qué tiene que hacer Colombia para que el fin del conflicto no se traduzca en la destrucción de su riqueza natural? Semana Sostenible.

ONU. (2014). Construcción de una paz territorial estable, duradera y sostenible en Colombia,. Bogotá: Sistema de las Naciones Unidas - Cooperaciòn Alemana,.

Osorio, A. R. (2003). Aproximaciones a los efectos ambientales, sociales y econónomicos de la erradicación de cultivos ilícitos para la aspersión aérea en Colombia. Agroalimentaria v.17.

Rodríguez, M. B. (2003). Los cultivos ilícitos y el medio ambiente. VIII Cátedra Anual de Historia "Ernesto Restrepo Tirado, Análisis histórico del narcotráfico en Colombia. Bogotá.

Romero M, C. E. (2008). Informe sobre el estado de la biodiversidad en Colombia 2006-2007. Instituto de Investigación Alexander Von Humboldt. Bogotá: Instituto de Investigación Alexander Von Humboldt.

Trejos, L. (2013). Aproximación a las dinámicas del crimen organizado en la ciudad de Barranquilla. El caso del Frente José Pablo Díaz de las Autodefensas Unidas de Colombia (200-2006). Justicia, 118-134. 
United Nations, O. (1976). Convention on the prohibition of use of environmental modification techniques for military purposes and other environmental purposes. Resolution 31/72. New York.

Vega, H. (2015). Aspectos dogmáticos y políticos criminales de la estructura general del delito en el sistema penal colombiano. Justicia, 27, 42-72. Recuperado de http://dx.doi.org/10.17081/just.3.27.320

VerdadAbierta.com. (s/f de s/f de s/f). La desmovilización: el proceso de paz (2003-2006). Verdad Abierta.

Villegas, M. G. (2010). Normas de papel. La cultura del incumplimiento de reglas. Bogotá- Colombia: Siglo del Hombre Editores.

Yáñez, M. (2013). El desplazamiento forzado en la jurisprudencia de la Corte Constitucional colombiana (1991-2003): momento previo a la declaratoria forma del estado de cosas inconstitucional. Revista Justicia, (23), 191-218. Barranquilla: Universidad Simón Bolívar 